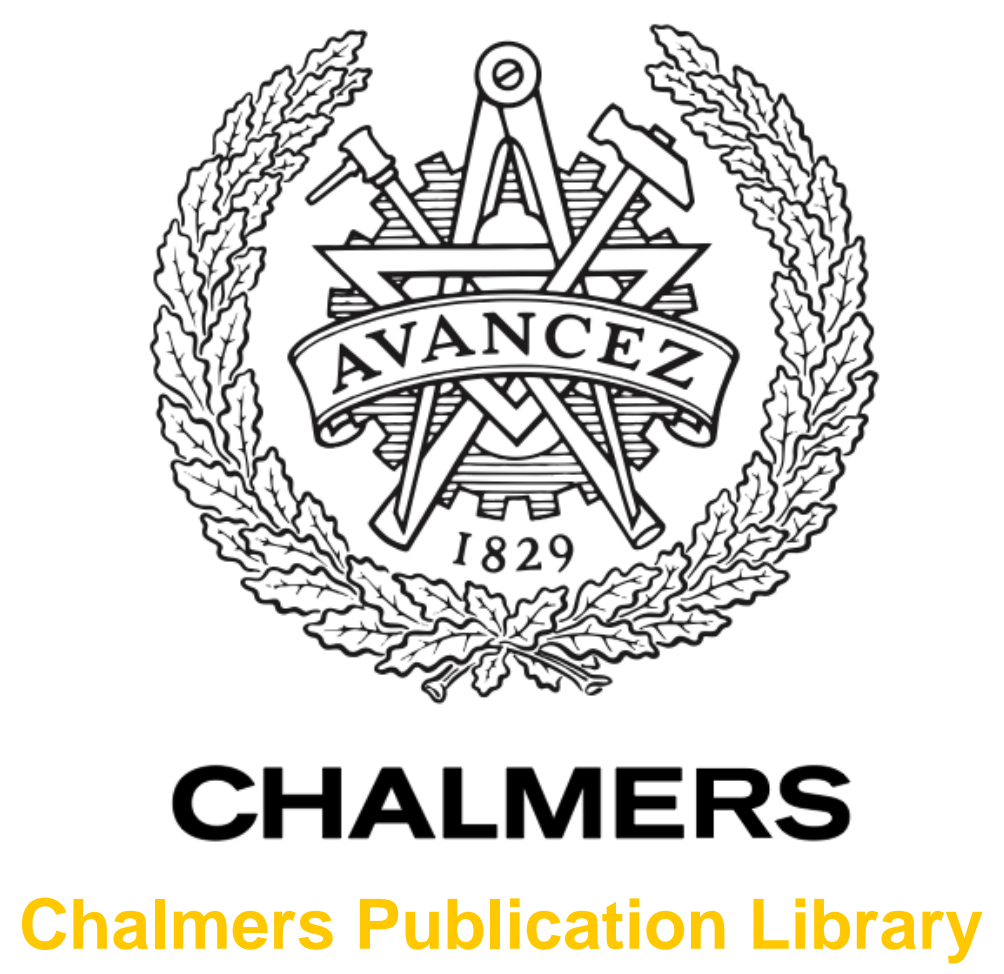

\title{
Model recovery anti-windup control for linear discrete time systems with magnitude and rate saturation
}

This document has been downloaded from Chalmers Publication Library (CPL). It is the author's version of a work that was accepted for publication in:

Proceedings of the American Control Conference (ISSN: 0743-1619)

Citation for the published paper:

Peni, T. ; Kulcsar, B. ; Bokor, J. (2012) "Model recovery anti-windup control for linear discrete time systems with magnitude and rate saturation". Proceedings of the American

Control Conference pp. 1543-1548.

Downloaded from: http://publications.lib.chalmers.se/publication/167053

Notice: Changes introduced as a result of publishing processes such as copy-editing and formatting may not be reflected in this document. For a definitive version of this work, please refer to the published source. Please note that access to the published version might require a subscription. 


\title{
Model recovery anti-windup control for linear discrete time systems with magnitude and rate saturation
}

\author{
T. Péni and B. Kulcsár and J. Bokor
}

\begin{abstract}
The paper proposes a model recovery anti-windup (MRAW) scheme for linear time-invariant and discrete-time systems under magnitude and rate saturation. The method is a modified, discrete-time counterpart of the algorithm presented in [4]. As it is usual in the MRAW framework the AW compensator contains the exact copy of the plant in order that the ideal (unsaturated) behavior can be preserved in the states. The compensator is a controller that aims to push the plant towards this intended behavior. The design of this control action can be reduced to a construction of a stabilizing state feedback acting on the saturated plant. In [4] this feedback is a linear one, which is designed by convex optimization by enlarging the ellipsoidal approximation of the invariant domain. This paper presents a different, set-theoretic approach, which is based on the precise construction of the maximal control invariant set. The proposed control is a nonlinear one generated by point wise convex optimization.
\end{abstract}

\section{INTRODUCTION}

Control input limitations are always present in real physical systems. If the controller is designed irrespective of these limitations the later appearance of a saturation may cause undesired behavior in the closed loop: it leads to performance degradation or even to instability. This effect is called controller windup.

One possible way to minimize the undesired effects of controller windup is using an anti-windup compensator. The concept is straightforward [8]: the controller is designed by ignoring the input nonlinearities and then a dynamic compensator is constructed, which modifies the inputs and outputs of the controller so that the following three criteria are fulfilled: (1) the closed loop is stable; (2) if there is no saturation the nominal performance is guaranteed; (3) in case of saturation the system is driven by the compensator so that the closed-loop signals return to the domain where they do not cause saturation and the nominal performance is recovered as quickly as possible.

The method presented in the paper belongs to the class of Model-Recovery Anti-Windup (MRAW) compensators. These approaches are common in that the dynamics of the compensator are the exact copy of the plant and the compensation is structured so that the difference

This work was supported by Control Engineering Research Group, Hungarian Academy of Sciences at the Budapest University of Technology and Economics.

T. Peni and J. Bokor are with Systems and Control Laboratory of Control and Automation Research Intitute of HAS, Kende 13-17, Budapest, Hungary. E-mail: ptescl.sztaki.hu

B. Kulcsár is with the Department of Signals and Systems, Chalmers University of Technology SE-412-96, Sweden. E-mail: kulcsar@chalmers.se. B. Kulcsár acknowledges the support of the Transport Area of Advance at Chalmers. between the states of the real plant and its copy holds all information about the ideal (saturation-free) behavior [6]. The compensator design generally boils down to a construction of a state feedback controller, which drives the plant's copy so that the real system tends towards the intended behavior. If there is no uncertainty in the system the MRAW compensator is totally independent of the actual controller, so it can work with any control method[4], [11]. Though all advantages of MRAW algorithms can be enjoyed only if the plant is precisely known, there are results for uncertain systems as well [10], [9].

This paper derives the discrete-time counterpart of the novel, MRAW compensator proposed for continuous time systems in [4]. In the paper cited two approaches (plantorder and extended dimensional) are discussed. They differ in the handling of the magnitude and rate limited saturation: the plant-order design considers the saturation as a single, dynamic nonlinearity while the extended model separates it into two (magnitude and rate) components. Depending on the open-loop properties of the plant three different anti-windup solutions are proposed for both methods. In this paper we focus only on the extended compensator structure with tradeoff solution, because it can be applied to open-loop unstable plants and enjoys some certain linearity properties.

Beside the precise formulation of the discrete-time compensation scheme the paper proposes a different, set-theoretic design method for the compensator stabilization. In [4] the saturation is inserted into the design framework by a modified sector condition, linear feedback is constructed and quadratic Lyapunov function is used to generate ellipsoidal approximation for the domain of applicability. In contrast we exploit the advantages of discrete-time formulation and characterize the guaranteed stability region as a polytopic set. Over this domain an optimization-based method is proposed, which can compute an admissible control input at every point of the entire region. The applicability of the presented method is demonstrated on a numerical example.

The paper is organized as follows. After the introduction and problem formulation the concept of model-recovery antiwindup is presented. Then, the discrete-time compensator structure is derived in sec IV. A separate section (sec. $\mathrm{V}$ ) is devoted to the design of compensator stabilization, because this is the point where the continuous-time procedure and its discrete-time version are conceptually differ. The paper concludes with a numerical example (sec. VI) and the planned future research (sec. VII). 


\section{NOTATION AND PRELIMINARIES}

This section summarizes the notation and the most important theorems used in the paper.

1) The notation is fairly standard. If $x$ is a vector then its $i$-th component is denoted by $x_{i}$. If $x$ is a vector valued signal generated by a discrete-time process, then $x$ (without superscript), $x^{+}$and $x^{-}$denote its value at the current, next and previous time steps, respectively.

2) The vector-valued saturation and dead-zone functions are denoted by $\operatorname{sat}_{R}(u)$ and $\mathrm{dz}_{R}(u)$ and are defined in the usual way: $\operatorname{sat}_{R}(u)_{i}=\max \left(\min \left(u_{i}, R_{i}\right)\right.$, $\left.-R_{i}\right), R_{i} \in \mathbb{R}, R_{i}>0$ and $\mathrm{dz}_{R}(u)=u-\operatorname{sat}_{R}(u)$.

3) The following theorem is taken from [4]. Given any pair $v, y \in \mathbb{R}$ and $\varepsilon \in(0,1)$, there exists $\epsilon \in[\varepsilon, 2-\varepsilon]$ s.t. the following equality holds:

$$
\operatorname{sat}_{S}(y+v)-y=\operatorname{sat}_{S \epsilon}(v)+\sigma
$$

where $|\sigma| \leq\left|2 \mathrm{dz}_{S}(1-\varepsilon)\right|$. The actual value of $\epsilon$ depends on the actual argument of the saturation function.

The following definitions and theorems can be found e.g. in [2]

4) A convex, compact set containing the origin in its interior is called $\mathrm{C}$-set. The Minkowski function of a C-set $\mathcal{S} \subseteq \mathbb{R}^{n}$ is defined for all $x \in \mathbb{R}^{n}$ as $\Psi(x)=$ $\inf \{\lambda \geq 0: x \in \lambda \mathcal{S}\}$.

5) A set is polyhedral if it is defined as an intersection of finite number of closed halfspaces. The polytope is a bounded polyhedral set. Polytopes and polyhedral sets can be given by a set of linear ineqaulities, defining the hyperplanes. E.g. $\mathcal{P}=\{x \mid H x \leq h\}$, where " $\leq$ " is meant element-wise.

6) Let $x^{+}=A x+B u$ be a discrete time system with state and input constraints $x \in \mathcal{X}, u \in \mathcal{U}$, where $\mathcal{X}, \mathcal{U}$ are polyhedral $\mathrm{C}$-sets. $\mathcal{P}$ is a maximal control invariant set (MCIS) contained in $\mathcal{X}$ if $\mathcal{P} \subseteq \mathcal{X}$ and for all $x \in \mathcal{P}$ there exist $u \in \mathcal{U}$ s.t. $A x+B u \in \mathcal{P}$. In case of LTI systems the MCIS is a polytope and can be computed efficiently by the backward computation method described in [2] and in section $\mathrm{V}$.

7) A set $\mathcal{S}$ is $\lambda$-contractive for system $x^{+}=A x+B u$ if there exists $u \in \mathcal{U}$ s.t. $\Psi(A x+B u) \leq \lambda$ for all $x \in$ $\mathcal{S}$. If $\mathcal{S}$ is $\lambda$-contractive then the Minkowski function $\Psi(x)$ is a control Lyapunov function inside $\mathcal{S}$ ([2]). The stabilizing control input associated with $\Psi(x)$ can be constructed by on-line optimization. The details of the algorithm can be found in [2] and in section V.

8) For some $\lambda>0$, the MCIS of $x^{+}=(A / \lambda) x+(B / \lambda) u$ is a $\lambda$-contractive set of $x^{+}=A x+B u$.

\section{PROBLEM FORMULATION}

The problem setup is similar to the one formulated in [4]. The system to be controlled is linear, time invariant and given in discrete-time state space form, as follows:

$$
\begin{aligned}
x^{+} & =A x+B_{u} u+B_{d} d \\
y & =C_{y} x+D_{y u} u+D_{y d} d \\
z & =C_{z} x+D_{z u} u+D_{z d} d
\end{aligned}
$$

where $x \in \mathbb{R}^{n}, u \in \mathbb{R}^{m}, y \in \mathbb{R}^{n_{y}}, z \in \mathbb{R}^{n_{z}}$ are the state, control input, measured output and performance output, respectively. (The plant may be open-loop exponentially unstable, as the method discussed in the paper does not restricted to stable systems.) We assume that an unconstrained controller has already been designed for the plant:

$$
\begin{aligned}
x_{c+} & =f\left(x_{c}, u_{c}, r\right) \\
y_{c} & =g\left(x_{c}, u_{c}, r\right)
\end{aligned}
$$

where $x_{c}, u_{c}, r, y_{c}$ are respectively the controller state, controller input, the reference to be tracked and the controller output. This general form is used to indicate that the controller can have arbitrary structure, even a nonlinear one. If there is no saturation, the loop is closed by the following interconnection:

$$
u=y_{c}, \quad u_{c}=y
$$

We assume that this nominal closed loop system (formed by (1)-(2)-(3)) is well posed, exponentially stable and all performance requirements prescribed for $z$ are satisfied for all possible disturbance and reference signals. This means that the nominal loop produces the intended behavior.

The saturated system is obtained from (1)-(2) by inserting the magnitude and rate limited actuator, defined as follows, between the controller output and plant input:

$$
u=\operatorname{sat}_{M R}(\nu)=u^{-}+\operatorname{sat}_{R}\left(\operatorname{sat}_{M}(\nu)-u^{-}\right)
$$

where

$$
\nu=y_{c}
$$

In (4) $M=\left[M_{1}, M_{2}, \ldots, M_{m}\right]$ and $R=\left[R_{1}, R_{2}, \ldots, R_{m}\right]$, $M_{i}, R_{i}>0$ denote the magnitude and rate limits. The output of (4) satisfies

$$
-M_{i} \leq u_{i} \leq M_{i}, \quad-R_{i} \leq u_{i}-u_{i}^{-} \leq R_{i}
$$

for all input $\nu$. Dynamics (4) is one possible model that is able to mimic the behavior of a rate and magnitude limited actuator. It can be verified that this discrete time model has the same advantageous properties than its continuous counterpart [5].

To distinguish the non-saturated system from the saturated one, the signals generated by (1)-(2)-(3) will be denoted by $\hat{\imath},(\hat{x}, \hat{z}$, etc. $)$

Our aim is to design an AW compensator to compensate the unintended effects of control input limitations caused by the saturating actuator (4).

\section{MODEL RECOVERY ANTI-WINDUP COMPENSATION FOR DISCRETE-TIME SYSTEMS}

In this section we derive the discrete-time counterpart of the extended, model-recovery anti-windup compensation proposed for continuous-time systems in [4].

As we have mentioned, the name "model-recovery" means that the compensator dynamics are the exact copy of the 
plant. In the extended structure the $n$ states given by the plant is extended with $m$ more state variables, each one corresponds to one rate limited input:

$$
\begin{aligned}
x_{a w}^{+} & =A x_{a w}+B_{u}\left(u-y_{c}\right) \\
\delta & =\operatorname{sat}_{R}\left(y_{c}-y_{c}^{-}+v_{1}^{-}\right)+\delta^{-} \\
y_{a w} & =C_{y} x_{a w}+D_{y u}\left(u-y_{c}\right) \\
z_{a w} & =C_{z} x_{a w}+D_{z u}\left(u-y_{c}\right)
\end{aligned}
$$

The compensator input $v_{1}$ will be determined later. The AW compensator is connected to the closed loop formed by (1)(2)-(4) in the following way:

$$
\nu=\operatorname{sat}_{M}(\delta), \quad u_{c}=y-y_{a w}
$$

The block-diagram of the entire structure is depicted in Figure 1. Consider now the dynamics of the controller and the state difference $x-x_{a w}$ :

$$
\begin{aligned}
x^{+}-x_{a w}^{+} & =A\left(x-x_{a w}\right)+B_{u} y_{c}+B_{d} d \\
y-y_{a w} & =C_{y}\left(x-x_{a w}\right)+D_{y d} d \\
z-z_{a w} & =C_{z}\left(x-x_{a w}\right)+D_{z u} y_{c}+D_{z d} d \\
x_{c}^{+} & =f\left(x_{c}, y-y_{a w}, r\right) \\
y_{c} & =g\left(x_{c}, y-y_{a w}, r\right)
\end{aligned}
$$

It is easy to see that (9)-(10) describes the same dynamics as (1)-(2)-(3), so in case of $\left(x-x_{a w}\right)(0)=x(0)$, (i.e. $x_{a w}(0)=$ $0)$ the trajectory of (9)-(10) coincides with the trajectory of (1)-(2)-(3), i.e.

$$
\begin{aligned}
y_{c}(k)=\hat{u}(k) & =\hat{y}_{c}(k), \\
\left(x-x_{a w}\right)(k) & =\hat{x}(k), \\
\left(z-z_{a w}\right)(k) & =\hat{z}(k)
\end{aligned}
$$

If the actuator saturates and consequently the state of the system start diverging from the ideal, unsaturated behavior, the state difference will carry the information how the ideal trajectory should look like. Exploiting this property, the saturation effect can be compensated and the ideal behavior can be recovered if $z$ is forced to converge to the ideal output $z-z_{a w}$, i.e. to make $z_{a w} \rightarrow 0$. This is the goal, which has to be achieved by suitably choosing the conditioning signal $v_{1}$.

To this end, we introduce first a new state variable $\delta_{a w}=$ $\delta-y_{c}$ which is defined to evolve according to the following dynamics:

$$
\begin{aligned}
\delta_{a w} & =\delta_{a w}^{-}+\left(\delta-\delta^{-}\right)-\left(y_{c}-y_{c}^{-}\right) \\
& =\delta_{a w}^{-}+\operatorname{sat}_{R}\left(y_{c}-y_{c}^{-}+v_{1}^{-}\right)-\left(y_{c}-y_{c}^{-}\right) \\
& =\delta_{a w}^{-}+\operatorname{sat}_{R \epsilon}\left(v_{1}^{-}\right)+\sigma_{R}
\end{aligned}
$$

where the last equality and the following upper bound are direct consequences of item 3 of section II:

$$
\left|\sigma_{R}\right| \leq\left|2 \mathrm{dz}_{R(1-\varepsilon)}\left(y_{c}-y_{c}^{-}\right)\right|=\left|2 \mathrm{dz}_{R(1-\varepsilon)}\left(\hat{y}_{c}-\hat{y}_{c}^{-}\right)\right|
$$

The input difference $u-y_{c}$ can also be rewritten in a similar way:

$$
\begin{aligned}
u-y_{c} & =\operatorname{sat}_{M R}\left(\operatorname{sat}_{M}(\delta)\right)-y_{c}=\operatorname{sat}_{M}(\delta)-y_{c} \\
& =\operatorname{sat}_{M}\left(\delta-y_{c}+y_{c}\right)-y_{c} \\
& =\operatorname{sat}_{M \epsilon}\left(\delta_{a w}\right)+\sigma_{M}
\end{aligned}
$$

where

$$
\left|\sigma_{M}\right| \leq\left|2 \mathrm{dz}_{R(1-\varepsilon)}\left(y_{c}\right)\right|=\left|2 \mathrm{dz}_{R(1-\varepsilon)}\left(\hat{y}_{c}\right)\right|
$$

Using the new state equations - (12) and (14) - the dynamics of the compensator can be expressed as

$$
\begin{aligned}
x_{a w}^{+} & =A x_{a w}+B_{u} \operatorname{sat}_{M \epsilon}\left(\delta_{a w}\right)+B_{u} \sigma_{M} \\
\delta_{a w}^{+} & =\delta_{a w}+\operatorname{sat}_{R \epsilon}\left(v_{1}\right)+\sigma_{R}^{+} \\
y_{a w} & =C_{y} x_{a w}+D_{y u} \operatorname{sat}_{M \epsilon}\left(\delta_{a w}\right)+D_{y u} \sigma_{M} \\
z_{a w} & =C_{z} x_{a w}+D_{z u} \operatorname{sat}_{M \epsilon}\left(\delta_{a w}\right)+D_{z u} \sigma_{M}
\end{aligned}
$$

Note that the external signals $\sigma_{M}$ and $\sigma_{R}^{+}$are upper bounded by the dead-zone function of the differences $\hat{u}-\operatorname{sat}_{M}(\hat{u})$ and $\left(\hat{u}^{+}-\hat{u}\right)-\operatorname{sat}_{R}\left(\hat{u}^{+}-\hat{u}\right)$, that is, they estimate the control input loss caused by the magnitude and rate limited actuator.

The goal is to design the compensator input $v_{1}$ so that the states of (16) remain close to zero if $\sigma_{M} \neq 0$ or $\sigma_{R}^{+} \neq 0$ and converges to zero at the largest available decay rate when $\sigma_{M}=\sigma_{R}^{+}=0$.

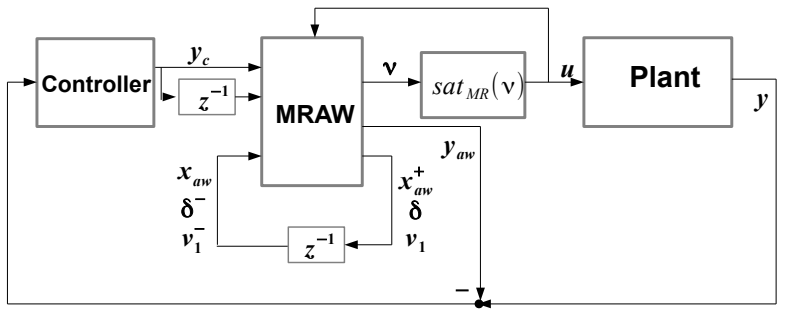

Fig. 1. The structure of discrete-time MRAW compensation.

\section{DESIGN OF COMPENSATOR INPUT}

Following the concept of [4] we can make the following assumption on the systems's behavior in saturating mode:

Assumption. The signals $\sigma_{M}, \sigma_{R}$ have compact support $[0, K], K \ll \infty$, that is $\sigma_{M}(k)=\sigma_{R}(k)=0$ for all $k>K$.

In other words, we assume that the system does not spend too much time in saturating mode. This assumption makes it possible to consider $\sigma_{M}, \sigma_{R}$ as short time perturbations, which push the state $\left(x_{a w}, \delta_{a w}\right)$ from 0 to some $\left(x_{a w}(K), \delta_{a w}(K)\right)$. If we neglect the short transient while the state reaches $\left(x_{a w}(K), \delta_{a w}(K)\right)$ it is enough to focus only on the time period $k>K$, i.e. when $\sigma_{M}, \sigma_{R}$ are zero again. The input $v_{1}$ has to be designed to steer the disturbance-free system back to the origin as quickly as possible. Since $v_{1}$ is constrained and $A$ can be unstable, this is possible only if $\left(x_{a w}(K), \delta_{a w}(K)\right)$ is in the maximal 
control invariant set of (16). Based on these observations, it makes sense to define $v_{1}$ as a suitable trade-off solution between the following two conditions:

- Make the domain of attraction as large as possible in order that the compensator converges for large values of $x_{a w}(K)$, i.e. the compensated loop can tolerate large input loss.

- Make also the decay rate as large as possible, for the unconstrained response can be recovered as fast as possible.

The point where the continuous and discrete time formulations significantly differ is the method chosen for constructing the feedback control $v_{1}$. In [4] $v_{1}$ is constructed in linear, state feedback form $v_{1}=K\left[\begin{array}{c}x_{a w} \\ \delta_{a w}\end{array}\right]$, the domain of attraction is estimated by the ellipsoidal level set of the quadratic Lyapunov function and the modified sector condition [7] is used to take the dead-zone nonlinearity into consideration through the LMI-based design procedure. For the discrete time case a different, set-theoretic approach is proposed in this paper. Starting from the saturation-free LTI system

$$
s^{+}=\left[\begin{array}{cc}
A & B_{u} \\
0 & I
\end{array}\right] s+\left[\begin{array}{l}
0 \\
I
\end{array}\right] v_{1}=A_{a w} s+B_{a w} v_{1}
$$

$\left(s \in \mathbb{R}^{n+m}\right)$, the saturation nonlinearities can be considered as linear state/input constraints

$$
\begin{array}{r}
\operatorname{sat}_{M \epsilon}\left(\delta_{a w}\right) \rightarrow\left|\delta_{a w}\right| \leq M \epsilon \\
\operatorname{sat}_{R \epsilon}\left(v_{1}\right) \rightarrow\left|v_{1}\right| \leq R \epsilon
\end{array}
$$

which have to be satisfied by (17). Then, picking an arbitrary $0<\lambda<1$ constant, we can construct the $\lambda$-contractive polytopic set $\mathcal{P}$ of (17), over which the Minkowski function serves as control Lyapunov function and the stabilizing $v_{1}$ can be computed at each point of $\mathcal{P}$ by on-line optimization (Item 7, Section II) ${ }^{1}$.

Applying item 8 of Section II the $\lambda$-contractive set of (17) can be determined by computing the MCIS of the scaled system

$$
s^{+}=\frac{1}{\lambda}\left[\begin{array}{cc}
A & B_{u} \\
0 & I
\end{array}\right] s+\frac{1}{\lambda}\left[\begin{array}{l}
0 \\
I
\end{array}\right] v_{1}=A_{a w, \lambda} s+B_{a w, \lambda} v_{1}
$$

Since the $\lambda$-contractive set converges to the MCIS of (17) as $\lambda$ tends to 1 , therefore the algorithm above enables us to extend to domain of attraction up to the MCIS, i.e. we are able to give state feedback control for all states, where it exists at all.

Going into the details, the first step of the controller design is the construction of the MCIS of (19). This step can be performed easily by using the following, backward computation method [2]:

\footnotetext{
${ }^{1}$ By definition or by construction all of the sets appearing in the paper contain the origin in the interior so every set is a C-set.
}

1) Let $\mathcal{P}_{0}=\left\{s \mid H_{0} s \leq h_{0}\right\} \subset \mathbb{R}^{n+m}$ be the initial polytope with

$$
H_{0}=\left[\begin{array}{cc}
I_{n} & 0 \\
-I_{n} & 0 \\
0 & I_{m} \\
0 & -I_{m}
\end{array}\right], \quad h_{0}=\left[\begin{array}{c}
x_{\max } \cdot 1_{n} \\
x_{\max } \cdot 1_{n} \\
M \epsilon \\
M \epsilon
\end{array}\right]
$$

where $x_{\max }$ is an arbitrary, positive value determining the domain of interest. It is used to render the initial set bounded. The constraints on $v_{1}$ define also a polytope, which is denoted by $\mathcal{U}=\left\{v_{1}|| v_{1} \mid \leq R \epsilon\right\}$.

Let $k=1$.

2) Compute $\overline{\mathcal{P}_{k}}$ from $\mathcal{P}_{k-1}=\left\{s \mid H_{k} s \leq h_{k}\right\}$ as follows:

$$
\begin{aligned}
\overline{\mathcal{P}_{k}:=} & \left\{\left(s, v_{1}\right) \mid s \in \mathcal{P}_{k-1}, v_{1} \in \mathcal{U},\right. \\
& {\left.\left[\begin{array}{ll}
H_{k-1} A_{a w, \lambda} & H_{k-1} B_{a w, \lambda}
\end{array}\right]\left[\begin{array}{c}
s \\
v_{1}
\end{array}\right] \leq h_{k-1}\right\} }
\end{aligned}
$$

3) $\mathcal{P}_{k}:=\operatorname{Proj}_{s} \overline{\mathcal{P}}_{k}$

4) If $\mathcal{P}_{k-1} \subset(1+\gamma) \mathcal{P}_{k}$ for some small $\gamma>0$ then STOP, otherwise $k:=k+1$ and GOTO 2 .

Remark. Although the value of $\epsilon$ depends on the actual argument of the corresponding saturation function, it is enough to perform the algorithm above with the smallest value $\epsilon=\varepsilon$, because it is the case where we get the most stringent constraints in (18).

If the algorithm terminates at step $k$, the set $\mathcal{P}_{k}$ will be a close approximation of the MCIS $\mathcal{P}$ of (19) and thus the $\lambda^{*}$-contractive set of (17). Here $\lambda^{*}$ is not equal to $\lambda$ because of the numerical approximation. Precisely, $\lambda^{*}=(1+\gamma) \lambda$. Having determined the contractive set it has to be stored because it is needed at each time step of control to compute the actual stabilizing compensator input $v_{1}$. If at time $k$ the compensator state is $s(k)=\left[x_{a w}(k) \delta_{a w}(k)\right]$ then $v_{1}(k)$ can be determined as a result of the following convex optimization problem [2]:

$$
v_{1}(k)=\arg \min \left\{\begin{array}{c}
\min _{v_{1}} \xi: \\
H\left(A_{a w} s+B_{a w} v_{1}\right) \leq \xi \cdot h \\
v_{1} \in \mathcal{U}, \xi>0
\end{array}\right.
$$

where $\mathcal{P}=\{s \mid H s \leq h\}$. The minimization of $\xi$ in (21) ensures that the control input will provide the largest possible decay rate at each time step. The stability of (17) under the control $v_{1}$ is certified by the Minkowski function, which is a Lyapunov function for the closed loop (17)-(21).

Remark. Although the applicability of $v_{1}$ generated by (21) is proved only for the case when $\sigma_{M}=\sigma_{R}^{+}=0$, the algorithm (21) can be applied in practice if $\sigma_{M} \neq 0$ or $\sigma_{R}^{+} \neq 0$. During this transient the state $\left(x_{a w}, \delta_{a w}\right)$ is not necessarily in $\mathcal{P}$ so (21) will terminate with $\xi>1$.

Remark. The trade-off between the size of the guaranteed stability domain and the rate of convergence can be controlled by $x_{\max }$, which determines the domain of interest. 
Larger $x_{\max }$ results in larger contractive set but produces smaller control inputs, while smaller $x_{\max }$ decreases the guaranteed stability domain and increases the rate of convergence.

\section{NUMERICAL EXAMPLE}

We consider the following two dimensional system, borrowed with slight modifications from the AWAST Toolbox [1]:

$$
\dot{x}=\left[\begin{array}{cc}
-0.5 & 1 \\
0.8 & -0.4
\end{array}\right] x+\left[\begin{array}{c}
-0.2 \\
-5
\end{array}\right] u, \quad y=x
$$

The system is discretized first with sampling time $T_{s}=0.1$. The discrete-time model corresponds to

$$
x^{+}=\left[\begin{array}{ll}
0.9550 & 0.0957 \\
0.0766 & 0.9646
\end{array}\right] x+\left[\begin{array}{l}
-0.0438 \\
-0.4916
\end{array}\right] u=A x+B_{u} u
$$

The first state variable $x_{1}$ has to track a reference value, therefore the following controller has been designed for the nominal plant:

$$
\begin{aligned}
x_{c}^{+} & =x_{c}+\left[\begin{array}{lll}
0.1 & -0.1 & 0
\end{array}\right]\left[\begin{array}{c}
r \\
u_{c}
\end{array}\right] \\
y_{c} & =-19.7698 x_{c}+\left[\begin{array}{lll}
0 & 8.0292 & 1.5811
\end{array}\right]\left[\begin{array}{c}
r \\
u_{c}
\end{array}\right]
\end{aligned}
$$

The closed loop behavior without input saturation is depicted in Figure 2. By inserting the actuator model (4) into the loop with limits $M=8, R=0.8$ the closed loop becomes unstable, as it can be seen in Figure 3. It is clear that the AW compensation is required to recover the stability and tracking performance. The compensator design starts with the MCIS algorithm. Setting $\lambda=0.95, x_{\max }=40$ and $\gamma=0.04$ we got the polytopic set depicted in Figure 4. It can be seen that the compensator is applicable over a large subset of states, i.e. it can tolerate relatively large input loss.

Figure 6 shows the simulation results with the AW compensation in the loop. The computation of $v_{1}$ by algorithm (21) takes approx. $0.04 \mathrm{~s}$ on $2.3 \mathrm{GHz}$, Intel i5 processor, so it works in real-time. As it is expected, the compensation slowed down the system but it managed to regain the stability.

\section{CONCLUSIONS AND FUTURE WORKS}

In the paper a model-reference anti-windup solution has been proposed for discrete-time linear systems. The algorithm follows the concept of the continuous time compensation proposed by [4]. The proposed method is a set-theoretic approach using fundamentally different tools than [4]. The drawback of the method is the increased computational time required by the on-line optimization at each time instant. The future research has to be directed towards eliminating this disadvantageous property. For this, multi-parametric linear programming can be applied which can provide all solutions of (21) as a piecewise linear function of the state ([3]).
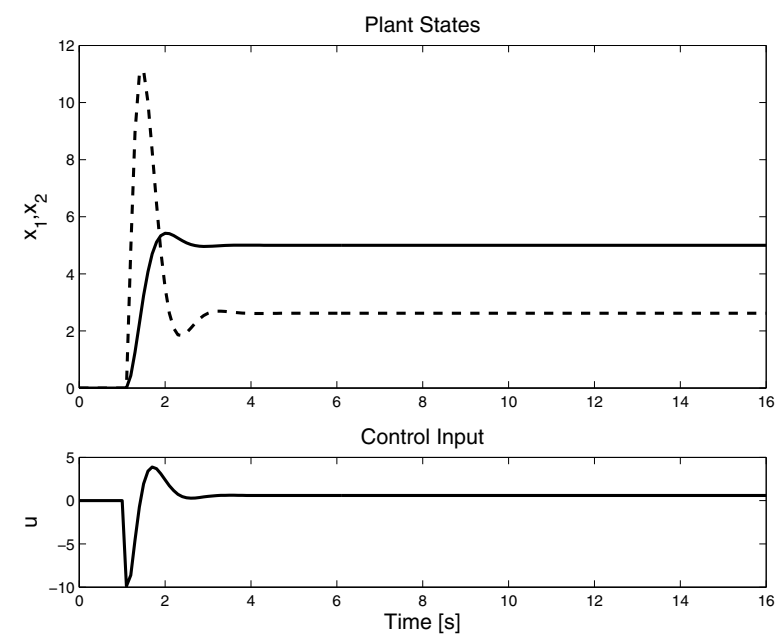

Fig. 2. Unsaturated system response. States: $x_{1}$-solid, $x_{2}$-dashed
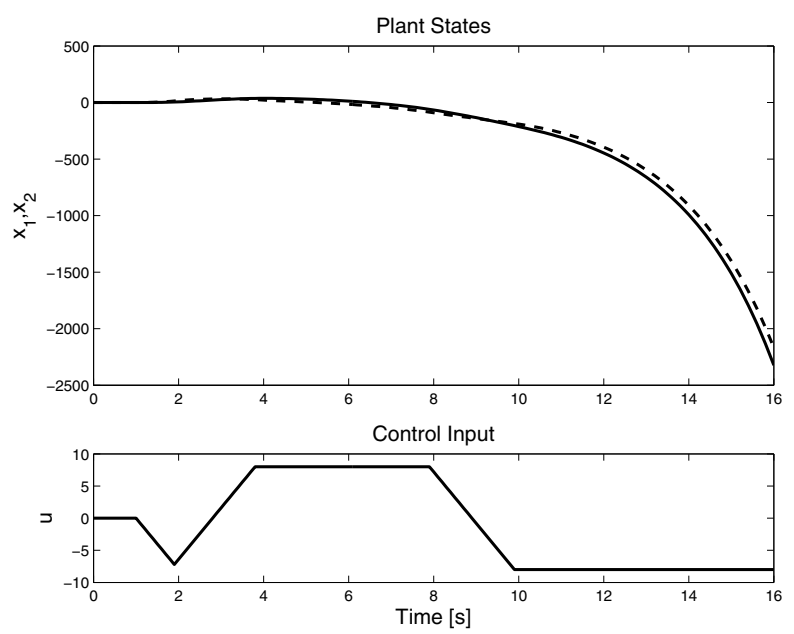

Fig. 3. System response with input saturation.

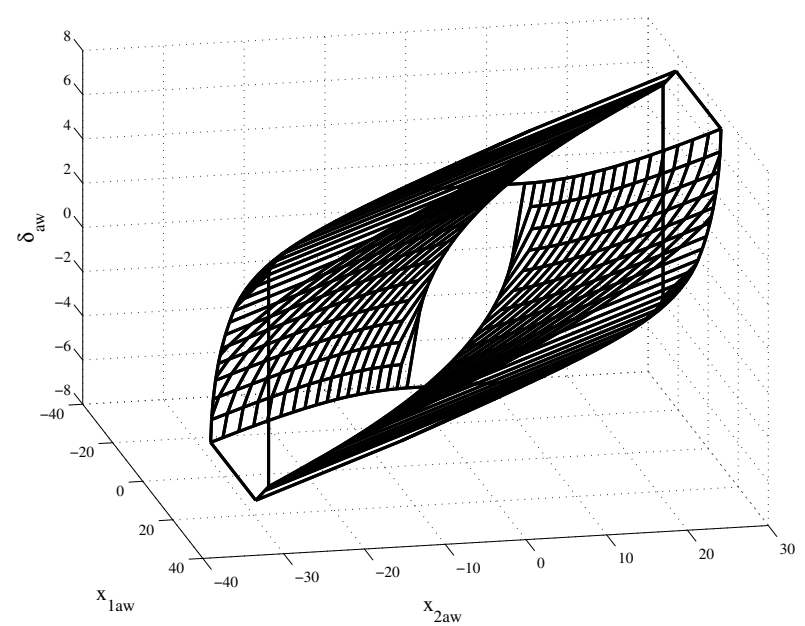

Fig. 4. The $\lambda$-contractive polytope computed for the AW compensator dynamics. 

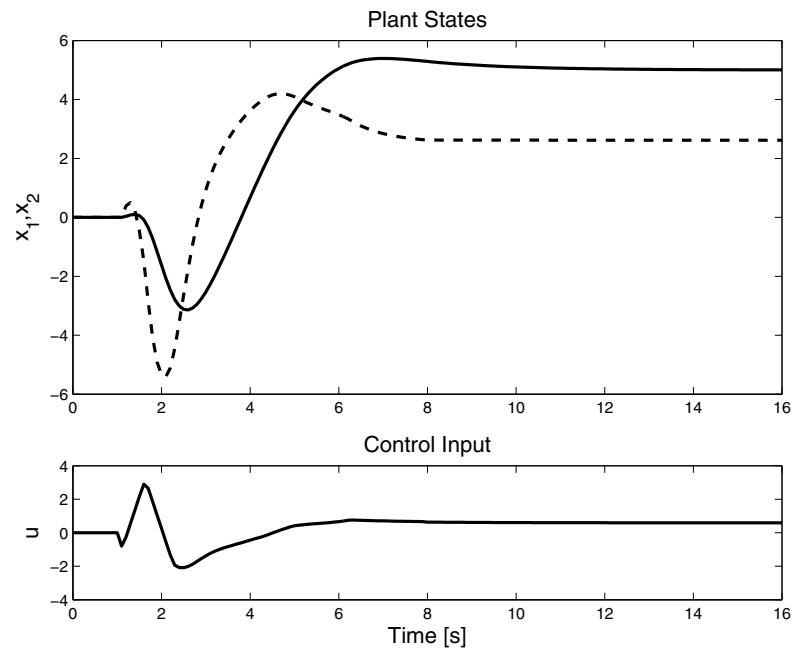

Fig. 5. System response with saturation and AW compensation in the loop. States: $x_{1}$ : solid, $x_{2}$ : dashed
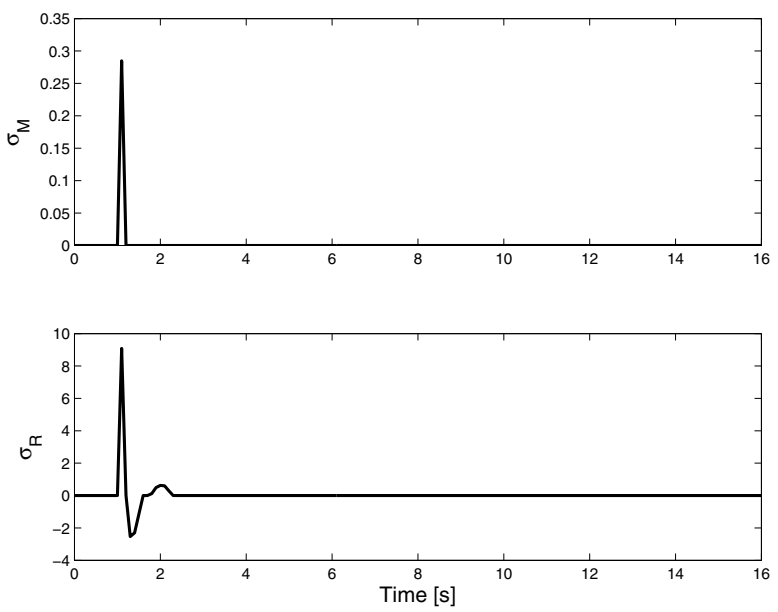

Fig. 6. Signals $\sigma_{M}$ and $\sigma_{R}$. They are short period impulses illustrating that our a-priori assumption on they time domain behavior was not far from reality.

\section{REFERENCES}

[1] J. M. Biannic and C. Roos. Introduction to AWAST : the anti-windup analysis and synthesis toolbox. In Computer-Aided Control Systems, pages $589-594,2008$.

[2] F. Blanchini and S. Miani. Set-Theoretic Methods in Control. Birkhauser, 2008.

[3] F. Borrelli. Constrained Optimal Control of Linear and Hybrid Systems, volume 290 of Lecture Notes in Control and Information Sciences. Springer, 2003.

[4] F. Forni, S. Galeani, and L. Zaccarian. Model recovery anti-windup for plants with rate and magnitude saturation. In Eurpean Control Conference (ECC), pages 324-329, 2009.

[5] S. Galeani, S. Onori, A.R.Teel, and L. Zaccarian. A magnitude and rate saturation model and its use in the solution of a static anti-windup problem. Systems \& Control Letters, 57:1-9, 2008.

[6] S. Galeani, S. Tarbouriech, M. Turner, and L. Zaccarian. A tutorial on modern anti-windup design. In European Control Conference (ECC), pages 306-323, 2009.

[7] J. M. Gomes and S. Tarbouriech. Antiwindup design with guaranteed regions of stability: An lmi-based approach. IEEE Transaction on Automatic Control, 50:106-111, 2005.

[8] M. V. Kothare, P. J. Campo, M. Morari, and C. N. Nett. A unified framework for the study of anti-windup designs. Automatica, 30(12):1869-1883, 1994.

[9] A. Marcos, M. C. Turner, and I. Postlethwaite. An architecture for design and analysis of high-performance robust antiwindup compensators. IEEE Transactions on Automatic Control, 52(9):1672-1679, 2007.

[10] M. C. Turner, G. Herrmann, and I. Postlethwaite. Accounting for uncertainty in anti-windup synthesis. In American Control Conference (ACC), pages 5292-5297, 2004.

[11] P. F. Weston and I. Postlethwaite. Linear conditioning for systems containing saturating actuators. Automatica, 36:1347-1354, 2000. 